\title{
3-I(2,4-dihydroxy-3,3-dimethylbutanoyl)-
} aminolpropanoic acid (Vitamin B5):

\section{Its Synthesis, Transformation into Coenzyme A and Role in Disease}

\section{MALIHA IMAMI}

Faculty of Arts and Science, University of Toronto, Canada

\section{Abstract}

This is a paper on Vitamin $B_{5}$, its role in coenzyme $A$ synthesis, and how a deficiency of $B_{5}$ can have catastrophic consequences on human health. In this review, the process of pantothenate synthesis in bacteria and plants will be explored, followed by the role of B5 in the synthesis of coenzyme A (CoA). Understanding these mechanisms is of critical importance to nutritional and clinical authorities to aid the formation of guidelines that promote good health and clinical treatment. The biosynthesis pathway of pantothenate in pathogenic bacteria is of particular interest in the future design of antibiotic drugs as it contains many potential enzyme targets that are absent and unnecessary in humans. The importance of $B_{5}$ and the CoA synthesis pathway is highlighted via a discussion of diseases that result, or are linked with, improper functioning of these systems. Subsequently, the paper explores the benefits of $B_{5}$ supplementation for cholesterol and inflammation reduction and immune response during tuberculosis infection. Lastly, the prevalence of poor vitamin $B_{5}$ intake in certain populations is discussed to highlight where $B_{5}$ consumption is increased and where supplementation should be promoted.

Keywords: pantothenic acid, vitamin supplementation, acetyl coenzyme A synthesis

\section{Introduction}

Vitamins are essential organic molecules required for a diverse array of physiological processes vital for organismal survival. For humans, there are 13 such vitamins that need to be acquired from dietary sources and failure to obtain the appropriate levels (both deficiencies and excesses) can have detrimental, or even fatal, effects on the human body (Zhang et. al., 2010). Across organisms, vitamins play a role in almost every biosynthetic pathway, metabolism, cell regulation and communication, and immune functioning and disease 
prevention (Lounder et. al., 2017; Ghanbari et al., 2016; Wong, 2017). Vitamins are classified into two categories based on their level of water solubility. Fat-soluble vitamins (such as vitamins $A, D, E$, and $K$ ) accumulate in tissues, while water-soluble vitamins (such as B-vitamins and vitamin C) are excreted and must be regularly replenished from dietary sources (Zhang et al., 2010). The class of B-vitamins has eight members and for the most part, these vitamins act as either coenzymes or as precursors of necessary coenzymes (Helliwell, 2017). In this review, the focus rests on vitamin $B_{5}$, also known as pantothenic acid.

Discovered in 1933 by Roger Williams (Burrows, 1936), $B_{5}$ is crucial for survival as it is indispensable to the synthesis of coenzyme A (CoA). CoA is involved in pyruvate oxidation following glycolysis in the citric acid cycle. Once vitamin $B_{5}$ (i.e. pantothenic acid) is converted into $\mathrm{CoA}$, it affects the allosteric regulation of enzymes involved in glucose catabolism, thereby governing the primary energy production process (Adina-Zada et. al., 2012; Pettit et al., 1975). Vitamin $B_{5}$ and CoA are also necessary for the synthesis of non-essential amino acids, fatty acids, glycoproteins, cholesterol, steroid hormones, membrane lipids, acetylcholine synthesis, and antibody generation (Rebeille et. al., 2008; Tahilliani \& Beinlich, 1991). Given the plethora of processes and uses of vitamin $B_{5}$, this review will focus on five facets of pantothenic acid research: (1) biosynthesis in bacteria and plants, (2) conversion into CoA, (3) the effects of abnormalities in these processes due to diseases, (4) the benefits of supplementation on cholesterol reduction, inflammation reduction, and immune system reduction, and (5) the prevalence of inadequate $B_{5}$ intake in the population.

Understanding the pathways via which pantothenic acid is synthesized and metabolized to $\mathrm{CoA}$ is of critical importance, given the substantive role of CoA in the maintenance of human health. This understanding can thereby shed light on potential health detriments that can arise out of $B_{5}$ deficiency and can inform regulatory health authorities on nutritional recommendations for the public. For example, knowledge of the $B_{5}$ synthesis pathway in bacteria allows for the identification of enzyme targets for antibiotics that are necessary to pathogen survival but are absent in healthy humans. Moreover, knowledge of how CoA is synthesized and problems in this pathway enable understanding of the etiology of certain neurodegenerative diseases and the causes of motor impairments in afflicted patients. Evidence of improved health via increased $B_{5}$ intake offers clear policy initiatives, which governmental authorities should prioritize in health recommendations to clinical and healthy populations. And the prevalence of 
deficiencies should be identified and quantified so that the problem is recognized and the affected populations can be instructed on how to improve their health and lead better lives.

\section{Vitamin $B_{5}$ : Sources, Structure and Significance}

In order to understand the multitude of roles it plays in the body, it is necessary to understand two features of vitamin $B_{5}$ : sources and structures. Nutritional authorities in the US (Food and Nutrition Board of the Institute of Medicine) recommend male and female adults consume $5 \mathrm{mg} /$ day and lower consumption levels for younger populations (Kelly, 2011). These nutritional recommendations are easily met as pantothenic acid is found in great abundance in many food sources (Kelly, 2011). Animal sources such as beef liver and kidney, lobster, egg yolk, shellfish, and chicken all have substantial amounts of vitamin $B_{5}(5 \mathrm{mg} / 100 \mathrm{~g}$ of food; Higdon et al., nd). Non-animal sources such as peanuts and peanut butter, almonds, wheat bran, and yeast also contain considerable amounts of vitamin B5 (average 2-3 mg/100g; Kelly, 2011; Higdon et al., nd). Additionally, many food sources, such as rice, are fortified with $B$ vitamins, including B5, which augments vitamin intake (Kyritsi et al., 2011). Unfortunately, industrial food processing, canning, freezing, and refining techniques result in a $31-75 \%$ loss of B5 content (Higdon et al., nd). The consequences of this loss are detailed in greater depth in later sections

The general structure of vitamin B5 is given in Figure 1. The vitamin exists in two enantiomeric forms, but only the $D$ enantiomer displays any biological activity in eukaryotes. As evident in Figure 1 , the pantothenic acid has pantoyl and $\beta$ alanine moieties (see Figure 1).

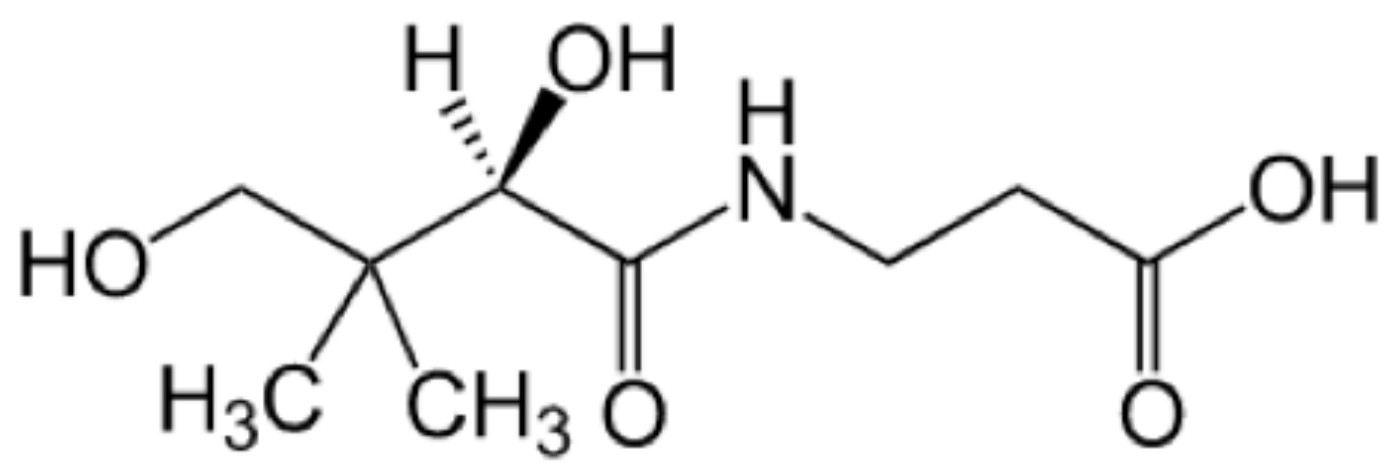

Figure 1. Chemical structure of pantothenic acid.

"Chemical structure of pantothenic acid (vitamin B5)" by Jü, CCO, via Wikimedia Commons 
The acid is synthesized in plants, archaea, and eubacteria by an enzyme-mediated pathway that combines the two aforementioned moieties (Villasenor et al., 2011; Webb et al., 2004). This pathway is a potentially rich area of research for the development of antibiotics and herbicides that are safe for humans as the enzyme targets of such treatments are not present in humans and most animals. The fundamental process of vitamin $B_{5}$ synthesis is in Leonardi and Jackowski (2007). The alanine moiety is formed from aspartate by an enzyme which is the product of the gene panD. The pantoyl moiety is formed by the enzymatic action of other gene products. ATP is then used for the condensation reaction that joins the pantoyl and alanine products via an enzyme coded by gene panC. (Leonardi \& Jackowski, 2007) panD activity is activated via another gene, panZ (Monteiro et. al., 2015). panZ is found in pathogens such as Salmonella enterica (Stuecker, Hodge \& Escalante-Semerena, 2012) and Yersinia pestis (Nozaki, Webb \& Niki, 2012). Research has shown that binding of compounds, such as pentyl pantothenamide, to pan $Z$ is a potent and novel way to inhibit $E$. coli growth and thus a promising area of antibiotic design and research to treat enteric bacterial infections (Leonardi \& Jackowski, 2007).

Animals lack the genomic sequence required for the enzymes involved in pantothenate synthesis and therefore must acquire pantothenic acid from the diet.
Once acquired, the vitamin is quickly metabolized into two main products: CoA and acyl carrier proteins (Kelly, 2011). CoA is an immensely important coenzyme that has critical roles in glucose catabolism, the citric acid cycle and ATP generation, fatty acid synthesis, cholesterol synthesis, phospholipid synthesis, pyrimidine base catabolism, and many other anabolic pathways. Acyl carrier proteins transfer acyl functional groups and are essential in the generation of synthase enzymes. While these pathways and mechanisms are clearly outside the scope of this paper, it is essential to list these roles in order to highlight the importance of their precursor, vitamin $B_{5}$.

The transformation of pantothenate into coenzyme A begins when a pantothenic acid kinase (pank1, panK 2, or pank 3 in humans; Yao et al., 2019) phosphorylates the pantoyl group of pantothenic acid by transferring the $y$-phosphate group from ATP (Higdon et al., nd). After a condensation reaction involving cytidine triphosphate, cysteine is added to the $\beta$ alanine group. A carboxyl group is removed to yield 4'-phosphopantetheine. ATP is used to add an adenosyl group to the 4' phosphate group. The sugar on the newly attached adenosyl group is phosphorylated once more to yield the final product, CoA. CoA and its acetylated form partake in the regulation of gene expression for the enzyme panK2 and are thus critical for the control of this process. Disruptions in feedback regulations lead to 
a variety of dysfunctions and diseases, which will be discussed in the subsequent section.

\section{Vitamin $B_{5}$ : Dysfunction, Disease and Deficiencies}

In this section, the diseases and effects of vitamin $B_{5}$ deficiencies are investigated. As described above, $B_{5}$ exerts most cellular effects via transformation into CoA and as such, this paper will first explore the results of improper regulation of $\mathrm{CoA}$ biosynthesis.

\section{PanK-Associated}

Regulation

and

Abnormalities. In $E$. coli, the mPanK1 $\beta$ controls the synthesis of the pank enzymes, and its in vitro expression was found to be stimulated by CoA, but inhibited by acetyl-CoA (Rock, Calder, Karim \& Jackwoski, 2000). Another study revealed that intracellular pantothenate kinases were only overexpressed if there was simultaneous supplementation with vitamin $B_{5}$ (Vadali, 2004). In a study of mice transfected with human panK2, overexpression of panK2 increased CoA levels in the skeletal muscles and was correlated with aberrant muscle function, reduced muscle size, and impaired mobility (Corbin et. al., 2017).

At the cellular level, the mitochondria of these mice had abnormal morphology, reduced ATP levels, and higher oxidative stress in muscle cells. As muscle cells typically have low levels of acetyl-CoA, and prior research demonstrated that acetyl-CoA is a strong inhibitor of panK gene expression, these findings demonstrate that overexpression of panK genes in presence of vitamin $B_{5}$ leads to increased $\mathrm{COA}$ and deleterious muscle development and impaired function. Without acetyl-CoA regulation of this system, skeletal muscles would deform.

Another enzyme-associated dysfunction related to CoA biosynthesis from vitamin $B_{5}$ is pantothenate kinase-associated neurodegeneration (PKAN), an autosomal recessive genetic disorder. PanK (the first enzyme in the synthetic pathway described above) mutations result in inactivated panK2 enzymes and the PKAN disease (Subramanian et al., 2020). A mouse model of this disease was found to have severe CoA deficiency despite the presence of $B_{5}$ precursors, and exhibited abnormal limb movement and extreme lethargy (Subramanian et al., 2020). In humans, PKAN presentations usually consist of dystonia, dysphagia, dementia, speech and psychological disturbances, neurological iron accumulation, and seizures (Subramanian et al., 2020; Hogarth et. al., 2017). In PKAN, the mutation of genes responsible for coding panK2 results in an absence of an enzyme to initiate the biosynthesis of CoA. As vitamin $B_{5}$ is the chemical scaffold of CoA, the vitamin was initially considered as a possible therapy for PKAN in order to increase substrate concentrations for whatever amount of pank molecules circulating in the 
system (Hogarth et. al., 2017). Some patients with atypical PKAN could potentially benefit from such vitamin supplementation treatment. However, no randomized and controlled clinical trial of this treatment has been conducted, despite current clinical guidelines for the treatment of PKAN, which include an extremely high dose of vitamin $B_{5}(250 \mathrm{mg}$ to as much as $5 \mathrm{~g}$ ) for 3 months. Such a guideline is based on anecdotal evidence from adults who perceive some benefit, but further research needs to be conducted with clinical populations of both atypical and classical PKAN (Hogarth et. al., 2017).

\section{Vitamin $B_{5}$-Related Deficiency and Diseases.}

PKAN shares a clinical feature with other neurodegenerative diseases, such as Huntington's, Parkinson's, and Alzheimer's disease: the accumulation of iron in the brain (Zhoue et. al., 2001). This shared feature revealed to clinicians and researchers that the $\mathrm{CoA}$ synthesis pathway may be associated with etiologies of other neurodegenerative diseases. Indeed, very recently, researchers have identified vitamin $B_{5}$ deficiency in the brains of deceased patients with Huntington's disease (HD). HD is an autosomal dominant disorder, where the patient experiences rapid and significant atrophy of cerebral tissues, ultimately culminating in severe physiological and cognitive decline and early death (Patassini et. al., 2019). A post-mortem examination of patients identified low cerebral vitamin $B_{5}$ ( $55 \%$ of $B_{5}$ levels in controls; Ismail et. al., 2020). As CoA and glucose metabolism are extremely vital for neural activity (Kennedy, 2016), the finding that $B_{5}$ level was so much lower in HD patients than in healthy controls indicates that this vitamin deficiency may play an important role in HD pathogenesis (Patassini et. al., 2019). Furthermore, the study also noted that areas where $B_{5}$ deficiency was high (e.g. basal ganglia) also exhibited greater atrophy. These findings suggest a possible malfunction in processes of $B_{5}$ uptake and absorption in HD. Specifically, this hints at a defect of the human sodium-dependent multivitamin transporter or the genes that encode it, in HD patients (Patassini et. al., 2019). Unfortunately, this study only collected post-mortem samples from 30 patients to uncover this correlation between $\mathrm{B}_{5}$ and HD. Further research with larger sample sizes and living patients is necessary to fortify such claims. The possibility of delaying HD-associated dementia by vitamin $B_{5}$ supplementation, as suggested by Patassini et al. (2019), also requires future research.

\section{$B_{5} \quad$ Increased Consumption and Supplementation Benefits}

It is evident that $B_{5}$ deficiencies are dangerous and associated with diseases in humans. On the other hand, higher intakes of $B_{5}$ have been associated with beneficial health outcomes. Three applications of $B_{5}$ to improve population health were suggested in research regarding 
cholesterol and inflammation reduction, and immune function improvement. Pantethine, a form of $B_{5}$ found in supplements has been shown to improve cardiovascular health via its cholesterolreducing capacity (Rumberger et. al., 2011). In a double-blind, randomized placebo-controlled trial (RCT), supplementation with pantethine for 12 weeks lowered low-density lipoproteincholesterol (LDL-C) and ameliorated the cardiovascular disease risk profile for 120 individuals in the trial (Rumberger et. al., 2011). The findings of this study were corroborated by another RCT, showing the robustness and generalizability of these findings (Evans et. al., 2014).

Another avenue of benefits from $B_{5}$ supplementation was discovered in the realm of inflammation reduction. Early atherosclerosis risk is exacerbated by underlying inflammation, and the risk is positively correlated with C-reactive protein (CRP) concentration (Jung et al., 2017). CRP binds to LDL-C and creates foam cell aggregates, a precursor for atherosclerosis (Jung et al., 2017). As mentioned before, $B_{5}$ supplementation can reduce LDL-C levels (Rumberger et al., 2011), but another mechanism through which the vitamin can confer protection from cardiovascular disease is its ability to reduce inflammation. Early investigations into this mechanism discovered that $B_{5}$ resulted in increased mitochondrial CoA, which was metabolized into glutathione (Slyshenkov et al., 2004; Demirci et al.,
2018). Researchers have postulated that the synthesis of glutathione resulted in reduced oxidative stress, thereby decreasing inflammation and biochemical markers of inflammation, such as CRP (Demirci et al., 2018). A long-term observational study on $B_{5}$ intake found that higher $B_{5}$ consumption was negatively correlated with CRP concentration with a small to moderate effect size (Demirci et al., 2018). While this correlation should not be confused with causation, future work on the benefits of $B_{5}$ supplementation for low to high-risk cardiovascular disease patients is warranted. Based on the above results, however, it appears that pantothenic acid supplementation offers protection against cardiovascular disease and could be a candidate for cardiovascular disease prevention therapeutic programs.

The third area of novel research with vitamin $B_{5}$ used mouse models infected with Mycobacterium tuberculosis. While the mechanisms via which vitamins improve immune function are still unclear, the effect of $B_{5}$ on inflammatory CRP suggests that it may play some role in modulating physiological responses to pathogens. $B_{5}$ was previously linked to increased expression of cytokines (protein-rich components of the immune system that are excreted by some immune cells to function in signalling and mediate response; Stephenson, 2001). $B_{5}$ was found to enhance the immune system's response (increased phagocytosis by macrophage) 
and reduce the number of tuberculosis colonies in the lungs of infected mice ( $\mathrm{He}$ et. al., 2018). $B_{5}$ was also shown to increase pro-inflammatory responses after infection but did not trigger excessive inflammation (Wilson et al., 2009). Currently, this line of research has not been extended to human tuberculosis patients and the exact mechanism via which $B_{5}$ exerts these protective effects against infection remains unclear. Further research on the role of $B_{5}$ in immune response and inflammatory signal cascades needs to be conducted.

\section{Deficiency Prevalence}

All the above studies suggest that $B_{5}$ supplementation incurs beneficial effects on consumers and that increasing consumption of $B_{5}$ is unlikely to cause harm. However, it is necessary to evaluate whether there are deficiencies in the larger population. Pantothenic acid deficiencies are rare as the vitamin is so prevalent in most food sources (Higdon et al., nd). However, modern industrial food processing and storage techniques might reduce nutrient content, and this may result in individuals with lower than the adequate intake of $B_{5}$. A 2010 study (using data from the Nation Health and Nutrition Survey) in Japan found that the majority of the sample had an adequate or near adequate intake of $B_{5}$ (Watanabe et. al., 2010). But a review of the other literature revealed that subsets of the global population had lower than recommended
$B_{5}$ intakes. In the United States, lower than recommended $B_{5}$ consumption was noted in adolescents (Eissenstat et al., 1986), children of low socioeconomic status in Texas (Wilson et al., 2009), nursing home residents (Wendland et al., 2003), and the elderly (Srinivasan, Christensen et al., 1981). As $B_{5}$ is synthesized by intestinal and gut bacteria, it is possible that low dietary intake can be compensated by this symbiotic relationship. This possibility needs to be evaluated in a clinical trial in greater detail. Regardless, given the possibility of differential nutritional profiles of different populations, health authorities should identify which groups are most at risk for deficiency and design an initiative to tackle such deficiencies. Indeed, if elderly and nursing home populations (where people with a higher risk of cardiovascular disease and infections reside in close proximity) have $B_{5}$ deficiencies, the studies discussed above suggest that these populations could benefit from supplementation to directly prevent high cholesterol, inflammation, and mount strong immune responses. 


\section{Conclusion}

Pantothenic acid is important for CoA synthesis and abnormalities in $B_{5}$ absorption and CoA synthesis has been implicated in many neurodegenerative diseases in humans, such as PKAN and Huntington's disease. Knowledge of these pathways has illuminated potential techniques for antibiotic design (by targeting pantothenate synthesis pathway of pathogens) and therapeutic interventions for patients. Further research needs to be done to determine conclusively the extent and nature of $B_{5}$ intake on improving health. Certain populations appear to be consuming inadequate amounts of $B_{5}$ and governmental health authorities should attempt to quantify the nature of this inadequate intake and enact nutritional guidelines and policies to correct such deficits.

\section{Note}

This paper was completed as part of a course at the University of Toronto Scarborough. 


\section{References:}

Adina-Zada, A., Zeczycki, T. N., St. Maurice, M., Jitrapakdee, S., Cleland, W. W., \& Attwood, P. V. (2012). Allosteric regulation of the biotin-dependent enzyme pyruvate carboxylase by acetyl-CoA. Biochemical Society Transactions, $567-572$. https://doi.org/10.1042/BST20120041

Burrows, W. (1936). The nutritional requirements of bacteria. The Quarterly Review of Biology, 11(4), $406-424$.

Corbin, D. R., Rehg, J. E., Shepherd, D. L., Stoilov, P., Percifield, R. J., Horner, L., Frase, S., Zhang, Y.-M., Rock, C. O., Hollander, J. M., Jackowski, S., \& Leonardi, R. (2017). Excess coenzyme A reduces skeletal muscle performance and strength in mice overexpressing human PANK2. Molecular genetics and metabolism, 120(4), $350-362$. https://doi.org/10.1016/j.ymgme.2017.02.001

Demirci, B., Yilmaz, M., \& Uysal, H. B. (2018). Protective effect of dexpanthenol (vitamin B5) in a rat model of LPS-induced endotoxic shock. Turkish Journal of Biochemistry, 43(6), 632637. https://doi.org/10.1515/tjb-2017-0321

Eissenstat, B. R., Wyse, B. W., \& Hansen, R. G. (1986). Pantothenic acid status of adolescents. The American Journal of Clinical Nutrition, 44(6), $931-937$. https://doi.org/10.1093/ajcn/44.6.931

Evans, M., Rumberger, J. A., Azumano, I., Napolitano, J. J., Citrolo, D., \& Kamiya, T. (2014). Pantethine, a derivative of vitamin B5, favorably alters total, LDL and non-HDL cholesterol in Low to moderate cardiovascular risk subjects eligible for statin therapy: A triple-blinded placebo and diet-controlled investigation. Vascular Health and Risk Management, 2014(10), 89-100. https://doi.org/10.2147/VHRM.S57116

Ghanbari, A. A., Shabani, K., \& Nejad, D. M. (2016). Protective effects of vitamin E consumption against $3 \mathrm{MT}$ electromagnetic field effects on oxidative parameters in substantia nigra in rats. Basic and Clinical Neuroscience, 7(4), $315-322$. http://dx.crossref.org/10.15412/J.BCN.03070404

He, W., Hu, S., Du, X., Wen, Q., Zhong, X. P., Zhou, X., Zhou, C., Xiong, W., Gao, Y., Zhang, S., Wang, R., Yang, J., \& Ma, L. (2018). Vitamin B5 reduces bacterial growth via regulating innate immunity and adaptive immunity in mice infected with Mycobacterium tuberculosis. Frontiers in Immunology, 9, Article 365. https://doi.org/10.3389/fimmu.2018.00365 
Helliwell, K. E. (2017). The roles of B vitamins in phytoplankton nutrition: new perspectives and prospects. New Phytologist, 216(1), 62-68. https://doi.org/10.1111/nph.14669

Higdon, J., Drake, V. J., \& Delage, B. (n.d.). Pantothenic Acid. Oregon State University. Retrieved March 16, 2020, from https://lpi.oregonstate.edu/mic/vitamins/pantothenic-acid

Hogarth, P., Kurian, M. A., Gregory, A., Csányi, B., Zagustin, T., Kmiec, T., Wood, P., Klucken, A., Scalise, N., Sofia, F., Klopstock, T., Zorzi, G., Nardocci, N., \& Hayflisk, S. J. (2017). Consensus clinical management guideline for pantothenate kinase-associated neurodegeneration (PKAN). Molecular Genetics and Metabolism, 120(3), 278-287. https://doi.org/10.1016/j.ymgme.2016.11.004

Ismail, N., Kureishy, N., Church, S. J., Scholefield, M., Unwin, R. D., Xu, J., Patassini, S., \& Cooper, G. J. S. (2020). Vitamin B5 (d-pantothenic acid) localizes in myelinated structures of the rat brain: Potential role for cerebral vitamin B5 stores in local myelin homeostasis. Biochemical and Biophysical Research Communications, 522(1), 220-225. https://doi.org/10.1016/j.bbrc.2019.11.052

Jung, S., Kim, M. K., \& Choi, B. Y. (2017). The long-term relationship between dietary pantothenic acid (vitamin B5) intake and C-reactive protein concentration in adults aged 40 years and older. Nutrition, Metabolism and Cardiovascular Diseases, 27(9), 806-816. https://doi.org/10.1016/j.numecd.2017.05.008

Kelly, G. S. (2011). Pantothenic acid. Alternative Medicine Review, 16(3), 263-275.

Kennedy, D. O. (2016). B vitamins and the brain: mechanisms, dose and efficacy-a review. Nutrients, 8(2), 68. https://doi.org/10.3390/nu8020068

Kyritsi, A., Tzia, C., \& Karathanos, V. T. (2011). Vitamin fortified rice grain using spraying and soaking methods. LWT - Food Science and Technology, 44(1), 312-320. https://doi.org/10.1016/j.lwt.2010.06.001

Leonardi, R., \& Jackowski, S. (2007). Biosynthesis of pantothenic acid and coenzyme A. EcoSal Plus, 2(2). https://doi.org/10.1128/ecosalplus.3.6.3.4

Lounder, D. T., Khandelwal, P., Dandoy, C. E., Jodele, S., Grimley, M. S., Wallace, G., Lane, A., Taggart, C., Teusink-Cross, A. C., Lake, K. E., \& Davies, S. M. (2017). Lower levels of vitamin A are associated with increased gastrointestinal graft-versus-host disease in children. Blood, 129(20), 2801-2807. https://doi.org/10.1182/blood-2017-02-765826 
Monteiro, D. C., Patel, V., Bartlett, C. P., Nozaki, S., Grant, T. D., Gowdy, J. A., Thompson, G. S., Kalverda, A. P., Snell, E. H., Niki, H., Pearson, A. R., \& Webb, M. E. (2015). The structure of the PanD/PanZ protein complex reveals negative feedback regulation of pantothenate biosynthesis by coenzyme A. Chemistry \& Biology, 22(4), 492-503. https://doi.org/10.1016/j.chembiol.2015.03.017

Nozaki, S., Webb, M. E., \& Niki, H. (2012). An activator for pyruvoyl-dependent l-aspartate adecarboxylase is conserved in a small group of the $y$-proteobacteria including Escherichia coli. MicrobiologyOpen, 1(3), 298-310. https://doi.org/10.1002/mbo3.34

Patassini, S., Begley, P., Xu, J., Church, S. J., Kureishy, N., Reid, S. J., Waldvogel, H. J., Faull, R. L. M., Snell, R. G., Unwin, R. D., \& Cooper, G. J. S. (2019). Cerebral Vitamin B5 (D-Pantothenic Acid) Deficiency as a Potential Cause of Metabolic Perturbation and Neurodegeneration in Huntington's Disease. Metabolites, 9(6), 113. https://doi.org/10.3390/metabo9060113

Pettit, F. H., Pelley, J. W., \& Reed, L. J. (1975). Regulation of pyruvate dehydrogenase kinase and phosphatase by acetyl-CoA/CoA and NADH/NAD ratios. Biochemical and biophysical research communications, 65(2), 575-582. https://doi.org/10.1016/S0006-291X(75)80185-9

Rébeillé, F., Ravanel, S., Marquet, A., Mendel, R. R., Smith, A. G., \& Warren, M. J. (2007). Roles of vitamins B5, B8, B9, B12 and molybdenum cofactor at cellular and organismal levels. Natural Product Reports, 24(5), 949-962. https://doi.org/10.1039/B703104C

Rock, C. O., Calder, R. B., Karim, M. A., \& Jackowski, S. (2000). Pantothenate kinase regulation of the intracellular concentration of coenzyme A. Journal of Biological Chemistry, 275(2), 1377-1383. https://doi.org/10.1074/jbc.275.2.1377

Rumberger, J. A., Napolitano, J., Azumano, I., Kamiya, T., \& Evans, M. (2011). Pantethine, a derivative of vitamin B5 used as a nutritional supplement, favourably alters low-density lipoprotein cholesterol metabolism in low-to moderate-cardiovascular risk North American subjects: a triple-blinded placebo and diet-controlled investigation. Nutrition Research, 31(8), 608-615. https://doi.org/10.1016/j.nutres.2011.08.001

Slyshenkov, V. S., Dymkowska, D., \& Wojtczak, L. (2004). Pantothenic acid and pantothenol increase biosynthesis of glutathione by boosting cell energetics. FEBS Letters, 569(1-3), 169172. https://doi.org/10.1016/j.febslet.2004.05.044 
Srinivasan, V., Christensen, N., Wyse, B. W., \& Hansen, R. G. (1981). Pantothenic acid nutritional status in the elderly-institutionalized and noninstitutionalized. The American Journal of Clinical Nutrition, 34(9), 1736-1742. https://doi.org/10.1093/ajcn/34.9.1736

Stephensen, C. B. (2001). Vitamin A, infection, and immune function. Annual Review of Nutrition, 21(1), $167-192$.

Stuecker, T. N., Hodge, K. M., \& Escalante-Semerena, J. C. (2012). The missing link in coenzyme A biosynthesis: PanM (formerly YhhK), a yeast GCN5 acetyltransferase homologue triggers aspartate decarboxylase (PanD) maturation in Salmonella enterica. Molecular Microbiology, 84(4), 608-619. https://doi.org/10.1111/j.1365-2958.2012.08046.x

Subramanian, C., Yao, J., Frank, M. W., Rock, C. O., \& Jackowski, S. (2020). A pantothenate kinase-deficient mouse model reveals a gene expression program associated with brain coenzyme a reduction. Biochimica et Biophysica Acta (BBA) - Molecular Basis of Disease, 1866(5), 165663. https://doi.org/10.1016/j.bbadis.2020.165663

Tahiliani, A. G., \& Beinlich, C. J. (1991). Pantothenic acid in health and disease. Vitamins \& Hormones, 46, 165-228. https://doi.org/10.1016/S0083-6729(08)60684-6

Vadali, R. V., Bennett, G. N., \& San, K. Y. (2004). Cofactor engineering of intracellular CoA/acetyl-CoA and its effect on metabolic flux redistribution in Escherichia coli. Metabolic Engineering, 6(2), 133-139. https://doi.org/10.1016/j.ymben.2004.02.001

Villaseñor, T., Brom, S., Dávalos, A., Lozano, L., Romero, D., \& García-de los Santos, A. (2011). Housekeeping genes essential for pantothenate biosynthesis are plasmid-encoded in Rhizobium etli and Rhizobium leguminosarum. BMC Microbiology, 11(1), 66. https://doi.org/10.1186/1471-2180-11-66

Walsh, J. H., Wyse, B. W., \& Hansen, G. (1981). Pantothenic acid content of a nursing home diet. Annals of Nutrition and Metabolism, 25(3), 178-181. https://doi.org/10.1159/000176492

Watanabe, T., Suemura, K., Taniguchi, A., Ebara, S., Kimura, S., \& Fukui, T. (2010). Dietary intake of seven B vitamins based on a total diet study in Japan. Journal of Nutritional Science and Vitaminology, 56(5), 279-286. https://doi.org/10.3177/jnsv.56.279

Webb, M. E., Smith, A. G., \& Abell, C. (2004). Biosynthesis of pantothenate. Natural Product Reports, 21(6), 695-721. https://doi.org/10.1039/B316419P 
Wendland, B. E., Greenwood, C. E., Weinberg, I., \& Young, K. W. (2003). Malnutrition in institutionalized seniors: The iatrogenic component. Journal of the American Geriatrics Society, 51(1), 85 -90. https://doi.org/10.1034/j.1601-5215.2002.51015.x

Wilson, T. A., Adolph, A. L., \& Butte, N. F. (2009). Nutrient adequacy and diet quality in nonoverweight and overweight Hispanic children of low socioeconomic status: The Viva la Familia Study. Journal of the American Dietetic Association, 109(6), 1012-1021. https://doi.org/10.1016/j.jada.2009.03.007

Wong, C. (2017). Iron deficiency anaemia. Paediatrics and Child Health, 27(11), 527-529. https://doi-org/10.1016/j.paed.2017.08.004

Yao, J., Subramanian, C., Rock, C. O., \& Jackowski, S. (2019). Human pantothenate kinase 4 is a pseudo-pantothenate kinase. Protein Science, 28(6), 1031-1047. https://doi.org/10.1002/pro.3611

Zhang, Y., Zhou, W. E., Yan, J. Q., Liu, M., Zhou, Y., Shen, X., Ma, Y., Feng, X., Yang, J., \& Li, G. (2018). A review of the extraction and determination methods of thirteen essential vitamins to the human body: An update from 2010. Molecules, 23(6), 1484. https://doi.org/10.3390/molecules23061484

Zhou, B., Westaway, S. K., Levinson, B., Johnson, M. A., Gitschier, J., \& Hayflick, S. J. (2001). A novel pantothenate kinase gene (PANK2) is defective in Hallervorden-Spatz syndrome. Nature Genetics, 28(4), 345-349. https://doi.org/10.1038/ng572 